Place of Death recorded following assessment and 65\% achieved this. Further qualitative evaluation by a local university will provide more detailed information regarding benefit.

The 'Palliative Care in Heart Failure' conference was held on 15 November 2018 with 51 attendees. There were speakers on a range of relevant topics (including external experts) and $98 \%$ of attendees rated it highly.

Conclusion This pilot has increased the number of patients with heart failure accessing hospice services, and by raising awareness of the needs of these patients will continue to do so.

\section{P-186 EVOLUTION - ADAPTING A FATIGUE AND BREATHLESSNESS COURSE FOR HEART FAILURE PATIENTS IN THE COMMUNITY}

'Dawn Tidmarsh, ${ }^{2}$ Emma Imam. ${ }^{1}$ Garden House Hospice Care, Letchworth, UK; ${ }^{2}$ Isabel Hospice, Welwyn Garden City, UK

\subsection{6/bmjspcare-2019-HUKNC.208}

Background and aim As part of a project to 'bridge the gap' between cardiology and palliative services for heart failure patients. The Living Well services in two partner hospices collaborated to design and deliver a heart failure specific fatigue and breathlessness course, aiming to educate patients to manage symptoms and improve quality of life through earlier engagement with hospice services. Led by two physiotherapists and supported by a Heart Failure Nurse Specialist, the course consisted of weekly tailored exercise (Taylor, 2019; NICE (NG106), 2018) an informative education session, relaxation and directed peer support.

Methods The course was modelled on the original Fatigue and Breathlessness courses (FAB) run at the hospices with exercises and education specifically modified for patients with heart failure. Education sessions addressed goal-setting and self-management, fatigue, breathlessness, eating, exercise, relaxation, advance care planning and living with heart failure. Attendees completed an IPOS assessment pre- and post- course as well as a Views on Care questionnaire. Therefore, quantitative and qualitative data was collected.

Results There were 25 referrals to the courses, 16 patients attended. There was an increase in the overall rating of quality of life post-attendance. $100 \%$ of respondents reported that the course gave them 'a lot of benefit' or 'some benefit'.

Qualitative data: what aspects of the course went well?

- Exercise circuit, good company

- Social side of the course. Learning breathing techniques

- Exercise information and meeting similar people

- Everything

- Exercise

- All of them.

Conclusions After attending the course, patients were equipped with the knowledge to manage their symptoms better. The patients reported enjoying the exercise and interaction with others. They now had the skills and confidence to exercise in the community and at home. At the end of the course many of the patients participated in further exercise and relaxation sessions within the hospices and the wider community.

\section{P-187 A REVOLUTION IN THE CARE OF HEART FAILURE PATIENTS}

${ }^{1}$ Ros Marvin, 'Sarah Bell, ${ }^{2}$ May Pheasant. 'Garden House Hospice Care, Letchworth Garden City, UKi ${ }^{2}$ sabel Hospice, Welwyn Garden City, UK

10.1136/bmjspcare-2019-HUKNC.209

Background Nearly a million people in the UK live with heart failure (HF) (Hospice UK, 2017). 30-40\% of patients die within a year of diagnosis, yet despite a high symptom burden they make up only around 4\% of those receiving specialist palliative care services (Hospice UK, 2017).

Two hospices working collaboratively received a grant from Hospice UK to bridge the gap between their care and the local NHS hospital trust cardiology team.

Aims To improve care for heart failure patients by:

- Providing a joined-up service for HF patients

- Developing a regional HF referral pathway

- Increasing hospice referrals

- Upskilling and educating the cardiology, specialist palliative care (SPC) and community healthcare teams

- Providing fatigue and breathlessness (FAB) courses.

Actions A consultant from one hospice and CNSs from the other hospice attended weekly hospital HF clinics and monthly multidisciplinary team meetings.

Hospices ran off-site, HF specific FAB courses.

A regional referral pathway between cardiology and SPC was developed.

Hospices held educational events for GPs and community healthcare professionals on HF management and palliation.

Outcomes Development of strong links between cardiology and SPC, increasing collaboration and knowledge within SPC and cardiology teams about the others roles.

HF referrals to the hospices doubled, with twice the national average of HF patients receiving hospice care. Patients accessed increased palliative support, holistic symptom management, advance care planning and family support.

Patient reported improvements in breathlessness, fatigue, weakness and quality of life following HF FAB courses.

Excellent feedback following community healthcare professionals' HF management and palliation education events.

Conclusion This collaborative project revolutionised the ongoing ability of cardiology and SPC to jointly manage HF patients' palliative care needs, resulting in a doubling in hospice referrals for HF patients. SPC support, including hospice referral and FAB courses, reduced HF patients' reported symptom burden. Community professionals' knowledge of the importance of SPC for HF patients was increased, supported by a comprehensive new referral pathway.

\section{P-188 IMPROVING ACCESS TO HOSPICE ENABLED CARE FOR HEART FAILURE PATIENTS - A SERVICE EVALUATION}

Tracy Livingstone, Jenny Welstand, Kay Ryan. Nightingale House Hospice, Wrexham, UK

10.1136/bmispcare-2019-HUKNC.210

In November 2017, following a successful application to St James's Place Charitable Foundation for funding, Nightingale House Hospice in partnership with Betsi Cadwaladr University Health Board, commenced an 18 month project to integrate 